Phys. cond. Matter 18, 179-187 (1974)

(C) by Springer-Verlag 1974

\title{
Theory of Transverse Neutron Inelastic Scattering in the Transition Metals
}

\author{
A. L. Kuzemsky \\ Laboratory of Theoretical Physics, Joint Institute for Nuclear Research, Moscow, USSR
}

Received July 6, 1974

A qualitative analysis of the transverse neutron inelastic cross section for the Hubbard model with $s-d$ hybridization in the framework of RPA is considered. The poles of the transverse generalized spin susceptibility are studied. It is found that the acoustic spin wave pole exists among the set of poles of dynamical spin susceptibility and that the non-interacting susceptibility determines the four quasi-Stoner continuum.

\section{Introduction}

The magnetic scattering of thermal neutrons is a technique of unique value for establishing both the static and the dynamic properties of magnetic correlations. It has already been extensively applied to the study of the static magnetic properties of the $3 d$ transition metals [1]. However, only in recent years, with the advent of more intense slow neutron sources and recent developments in the experimental technique, it has become possible to measure the long-wave-length inelastic scattering generally. Such experiments have provided considerable information about dynamical magnetic properties of the transition $3 d$ metals $[1-7]$.

Spin-only neutron scattering cross section for band $d$-electrons in transition metals is expressed in terms of generalized spin susceptibility, which measures the response of electrons to an external perturbation that couples to their spins, i.e., it describes electron spin dynamics. The poles of the generalized spin susceptibility functions determine the energy spectra of the excitations in the system. The explicit expressions for the poles are strongly dependent on the model used for the system and the character of employing approximations. Theoretical calculations of the generalized spin susceptibility in transition $3 d$ metals have been largely based on the single $d$-band Hubbard Hamiltonian and the most used approach to the interaction is still the Hartree-Fock or random-phase approximation. However, it is still unclear whether this model ever in fact exhibits ferromagnetism and, in particular, whether the ground state is ever the one of complete spin alinement. Also, there are certain aspects of the neutron inelastic scattering experiments in the transition $3 d$ metals that do not agree with such a simplified treatment [2-8].

Therefore, several calculations of the generalized spin susceptibility of the transition $3 d$ metals using various extensions of the Hubbard Hamiltonian have been published recently. These considerations take into account the multiband structure and orbital degeneracy $[8,9]$; the electron-phonon interaction $[10]$; the 
$s-d$ hybridization [11]; the interatomic interaction [12] and combined effects [13]. Direct study of generalized spin susceptibility in the neutron scattering experiments should be capable of providing important tests of theoretical models of magnetic transition $3 d$ metals.

In this paper we present within the framework of RPA a qualitative consideration of the transverse inelastic neutron cross section for the Hubbard model of magnetic $d$ electrons weakly hybridized with $s$-like conduction electrons. For this model the poles of the dynamical transverse susceptibility are studied. The oneparticle properties for various modifications of this model and its detailed foundation have been discussed in papers [14-16]. Recently Manochar [11] has discussed the spin-wave pole calculation for this model in the atomic limit for $d$-electrons and for the case of very strong $s-d$ hybridization. It seems, that these approximations are not appropriate to transition $3 d$ metals [14-16]. Our consideration based on the treatment Izuyama et al. [1] and the spin-flip excitation spectra of our system is derived as a natural extension of their calculations.

\section{Model Hamiltonian and the Formulation of the Problem}

The transition metal is described by a narrow $d$-band, with intraatomic Coulomb repulsion, a broad $s$-like conduction band and a $s-d$ mixing term coupling the two former terms. In the reciprocal-space representation the model Hamiltonian reads $[14-16]$

$$
H=H_{d}+H_{s}+H_{s-a}
$$

where

$$
H_{d}=\sum_{k, \sigma} E(k) d_{k \sigma}^{+} d_{k \sigma}+U / 2 N \sum_{\sigma} \sum_{k, k^{\prime}, q} d_{k+q, \sigma}^{+} d_{k, \sigma} d_{k^{\prime}-q,-\sigma}^{+} d_{k^{\prime},-\sigma}
$$

is the well-known Hubbard Hamiltonian [1].

$$
H_{s}=\sum_{k, \sigma} \varepsilon_{k} a_{k \sigma}^{+} a_{k \sigma}
$$

is the Hamiltonian of a broad $s$-like conduction band.

$$
H_{s-a}=\sum_{k, \sigma}\left(V_{k} a_{k \sigma}^{+} d_{k \sigma}+V_{k}^{*} d_{k \sigma}^{+} a_{k \sigma}\right)
$$

is the one-body operator which represents a mixture of the $d$-band and $s$-band electrons. The model Hamiltonian (2.1) incorporates the ideas of recent methods for calculating the electronic band structure for transition and noble metals and can be interpreted in terms of a series of Anderson "impurities" placed regularly in each site.

The magnetic scattering of unpolarized neutrons by an electronic system contains both spin and orbital terms. The spin-only part of the transverse neutron scattering cross section by the $d$-electronic system of the transition metal is given by the expression [1]

$$
\begin{aligned}
\left(\frac{d^{2} \sigma}{d \Omega d E^{\prime}}\right)_{\mathrm{tr} .}= & \left(\frac{\gamma e^{2}}{m_{e} c^{2}}\right)^{2}|F(q)|^{2} \frac{k^{\prime}}{k}\left(1+\tilde{q}_{z}^{2}\right) \times \\
& \times\left(-\frac{1}{2 \hbar}\right) \frac{e^{\hbar \omega \beta}}{e^{\hbar \omega \beta}-1}\left\{\operatorname{Im} G_{q}^{+}(\omega)+\operatorname{Im} G_{-q}^{-}(\omega)\right\}
\end{aligned}
$$


where

$$
G_{q}^{v}(t)=-i \theta(t)\left\langle\left[\tilde{s}^{v}(q, t), \tilde{s}^{-\nu}(q, 0)\right]-\right\rangle, \quad v= \pm
$$

is the two-time thermal retarded Green functions. It is convenient to express $G$ in terms of the generalized susceptibility:

$$
\frac{N}{\left(g \mu_{\mathrm{B}}\right)^{2}} X(q, \omega)=-\frac{2 \pi}{\hbar} G_{q}(\omega)=\left(-\frac{2 \pi}{\hbar}\right) \frac{1}{2 \pi} \int_{-\infty}^{\infty} d t e^{i \omega t} G_{q}(t) .
$$

Here $s(q)$ is the Fourier transform of the spin operator $s(r)$. With the aid of the definition $n(\omega)=[\exp (\beta \hbar \omega)-1]^{-1}$, the transverse scattering cross section (2.5) can be shown to reduce to

$$
\begin{aligned}
\left(\frac{d^{2} \sigma}{d \Omega d E^{\prime}}\right)_{\mathrm{tr} .} & =\left(\frac{\gamma e^{2}}{m_{e} c^{2}}\right)^{2}|F(q)|^{2} \frac{k^{\prime}}{k}\left(1+\tilde{q}_{z}^{2}\right) \times \\
& \times\left(-\frac{1}{2 \hbar}\right)\left\{(n(\omega)+1) \operatorname{Im} G_{-q}^{-}(\omega)+n(-\omega) \operatorname{Im} G_{q}^{-}(-\omega)\right\} .
\end{aligned}
$$

The remainder of this paper will be devoted to a calculation of this transverse scattering cross section within a Green function formalism.

\section{Approximate Calculation of the Reduced Susceptibility}

As is shown by Izuyama et al., provided that overlap integrals between Wannier functions on different sites are neglected, $X^{-}(q, \omega)$ may, in the tight-binding approximation for a single band Hubbard-Hamiltonian model, be calculated in terms of the Hartree-Fock magnetic response function $X^{H F}(q, \omega)[1]$. Now let us calculate $X^{-}(q, \omega)$ for the Hamiltonian (2.1). Thus the object now is to calculate the Green function $\ll \theta_{k}(q)=d_{k+q \downarrow}^{+} d_{k \uparrow} \mid B \gg{ }_{0}$. In the random phase approximation, the equations of motion for the Green functions are reduced to the closed simple form

1) $\left(\hbar \omega+\widetilde{E}_{\uparrow}(k+q)-\widetilde{E}_{\downarrow}(k)\right) \ll \theta_{k}(q) \mid B \gg \omega=\frac{\hbar}{2 \pi}\left(n_{k+q \downarrow}-n_{k \uparrow}\right) A(q, \omega)-$

$$
-V_{k+q} \ll a_{k+q_{\downarrow}}^{+} d_{k \uparrow}\left|B \gg_{\omega}+V_{k}^{*} \ll d_{k+q \downarrow}^{+} a_{k \dagger}\right| B \gg_{\omega} .
$$

2) $\left(\hbar \omega-\tilde{E}_{\downarrow}(k)+\varepsilon_{k+q}\right) \ll a_{k+q \downarrow}^{+} d_{k \downarrow} \mid B \gg \omega=\frac{\hbar}{2 \pi}\left\langle a_{k+q_{\downarrow}}^{+} d_{k+q \downarrow}\right\rangle A(q, \omega)-$

$$
-V_{k+q}^{*} \ll \theta_{k}(q)\left|B \gg_{\omega}+V_{k}^{*} \ll a_{k+q}^{+} a_{k \uparrow}\right| B \gg_{\omega} .
$$

3) $\quad\left(\hbar \omega-\widetilde{E}_{\uparrow}(k+q)-\varepsilon_{k}\right) \ll d_{k+q \downarrow}^{+} a_{k \uparrow} \mid B \gg_{\omega}=-\frac{\hbar}{2 \pi}\left\langle d_{k \uparrow}^{+} a_{k \uparrow}\right\rangle A(q, \omega)-$

$$
-V_{k+q} \ll a_{k+q \downarrow}^{+} a_{k \uparrow}\left|B \gg \omega+V_{k} \ll d_{k+q \downarrow}^{+} d_{k \uparrow}\right| B \gg \omega .
$$

4) $\left(\hbar \omega+\varepsilon_{k+q}-\varepsilon_{k}\right) \ll a_{k+q \downarrow}^{+} a_{k \uparrow} \mid B \gg_{\omega}=$

$$
=-V_{k+q}^{*} \ll d_{k+q \downarrow}^{+} a_{k \uparrow}\left|B \gg_{\omega}+V_{k} \ll a_{k+q \downarrow} d_{k \uparrow}\right| B \gg_{\omega} .
$$

Here the following definitions were introduced

$$
B=\sum_{k} d_{k \uparrow}^{+} d_{k+q \downarrow}
$$




$$
\begin{aligned}
\widetilde{E}_{\sigma}(k) & =E(k)+U(1 / N) \sum_{p} n_{p \sigma}, \\
A(q, \omega) & =1-(2 \pi / \hbar) U(1 / N) G_{q}^{-}(\omega), \\
\Delta & =U(1 / N) \sum_{p}\left(n_{p \downarrow}-n_{p \uparrow}\right) .
\end{aligned}
$$

To truncate the infinite hierarchy of Green function equations the RPA decoupling scheme (due to Izuyama et al.) is employed [1]

$$
\left[\theta_{k}(q), H_{d}\right]-\approx(E(k)-E(k+q)) \theta_{k}(q)+\Delta \theta_{k}(q)-U / N \sum_{p}\left(n_{k+q \downarrow}-n_{k \uparrow}\right) \theta_{p}(q) .
$$

$$
\begin{aligned}
{\left[d_{k+q \downarrow}^{+} a_{k \uparrow}, H_{a}\right] \approx-E(k+q) d_{k+q \downarrow}^{+} a_{k \uparrow}-U / N \sum_{p} n_{p \uparrow} d_{k+q \downarrow}^{+} a_{k \uparrow}+} \\
+U / N\left\langle d_{k \uparrow}^{+} a_{k \uparrow}\right\rangle \sum_{p} d_{p+q \downarrow}^{+} d_{p \uparrow}
\end{aligned}
$$

The mixing correlation functions are included in Eqs. (3.2), (3.3), (3.7) because in the region where the conduction band crosses the $d$-band the quasi-particle band structure will be critically dependent on the amount of $s-d$ mixing and the quantities mentioned will not be negligible [16]. Hence these terms must be retained. Now, we will use Eqs. (2.7) and (3.1) - (3.7) to determine the reduced transverse spin susceptibility in the RPA. It can be shown that

$$
X^{-}(q, \omega)=X^{\mathrm{HF}}(q, \omega)\left\{1-U /\left(g \mu_{\mathrm{B}}\right)^{2} \cdot X^{\mathrm{HF}}(q, \omega)\right\}^{-1},
$$

where

$$
\begin{aligned}
X^{\mathrm{HF}} & (q, \omega)=-\left(g \mu_{\mathrm{B}}\right)^{2} / N \sum_{k}\left\{( n _ { k + q \downarrow } - n _ { k \uparrow } ) \left[-\left|V_{k}\right|^{2}\left(\left(\hbar \omega+\widetilde{E}_{\downarrow}(k)+\varepsilon_{k+q}\right)+\right.\right.\right. \\
& \left.+\left(\hbar \omega+\widetilde{E}_{\uparrow}(k+q)-\varepsilon_{k}\right)\right)+\left(\hbar \omega+\varepsilon_{k+q}-\varepsilon_{k}\right)\left(\hbar \omega+\widetilde{E}_{\uparrow}(k+q)-\varepsilon_{k}\right) \times \\
& \left.\times\left(\hbar \omega-\widetilde{E}_{\downarrow}(k)+\varepsilon_{k+q}\right)\right]-\left(\hbar \omega+\varepsilon_{k+q}-\varepsilon_{k}\right)\left[V_{k}^{*}\left(\hbar \omega-\widetilde{E}_{\downarrow}(k)-\varepsilon_{k+q}\right) \times\right. \\
& \left.\left.\times\left\langle d_{k \uparrow}^{+} a_{k \uparrow}\right\rangle+V_{k}\left\langle a_{k+q \downarrow}^{+} d_{k+q \downarrow}\right\rangle\left(\hbar \omega+\widetilde{E}_{\uparrow}(k+q)-\varepsilon_{k}\right)\right]\right\} \times \\
& \times\left\{-\left|V_{k}\right|^{2}\left[\left(\hbar \omega+\widetilde{E}_{\uparrow}(k+q)-\widetilde{E}_{\downarrow}(k)\right)\left(\hbar \omega+\widetilde{E}_{\uparrow}(k+q)-\varepsilon_{k}\right)+\right.\right. \\
& +\left(\hbar \omega-\widetilde{E}_{\downarrow}(k)-\varepsilon_{k+q}\right)\left(\hbar \omega+\varepsilon_{k+q}-\varepsilon_{k}\right)+\left(\hbar \omega+\widetilde{E}_{\uparrow}(k+q)-\widetilde{E}_{\downarrow}(k)\right) \times \\
& \left.\times\left(\hbar \omega-\widetilde{E}_{\downarrow}(k)+\varepsilon_{k+q}\right)+\left(\hbar \omega+\widetilde{E}_{\uparrow}(k+q)-\varepsilon_{k}\right)\left(\hbar \omega+\varepsilon_{k+q}-\varepsilon_{k}\right)\right]+ \\
& +\left(\hbar \omega+\tilde{E}_{\uparrow}(k+q)-\tilde{E}_{\downarrow}(k)\right)\left(\hbar \omega-\widetilde{E}_{\downarrow}(k)+\varepsilon_{k+q}\right)\left(\hbar \omega+\widetilde{E}_{\uparrow}(k+q)-\varepsilon_{k}\right) \times \\
& \left.\times\left(\hbar \omega+\varepsilon_{k+q}-\varepsilon_{k}\right)\right\}^{-1}
\end{aligned}
$$

is the Hartree-Fock susceptibility. Note, that if $V_{k}=0$ then, $X^{\mathrm{HF}}(q, \omega)$ is reduced to

$$
X^{H \mathrm{~F}}(q, \omega)=\left(g \mu_{\mathrm{B}}\right)^{2} / N \sum_{k} \frac{n_{k \uparrow}-n_{k+q \downarrow}}{\hbar \omega+E(k+q)-E(k)-\Delta} .
$$

To evaluate $X^{\mathrm{HF}}(q, \omega)$ appearing in Eq. (3.8) we used the following additional approximation

$$
V_{k+q} \approx V_{k}
$$

which is valid in the long-wavelength limit $q \rightarrow 0$. The poles of the full susceptibility (3.8) corresponds to individual modes or the Stoner excitations and the spin-wave modes. The solutions corresponding to the individual spin-flip modes 
we shall consider in the next chapter. Now let us discuss the question about the existence of a spin-wave pole among the set of poles of the susceptibility (3.8). If we set $q=0$ in Eq. (3.8) the secular equation for poles becomes

$$
\begin{aligned}
1= & U / N \sum_{k}\left\{( n _ { k \uparrow } - n _ { k \downarrow } ) \left[-\left|V_{k}\right|^{2}(2 \hbar \omega-\Delta)+\hbar \omega\left(\hbar \omega+\widetilde{E}_{\uparrow}(k)-\varepsilon_{k}\right) \times\right.\right. \\
& \left.\times\left(\hbar \omega-\widetilde{E}_{\downarrow}(k)+\varepsilon_{k}\right)\right]-\hbar \omega\left[V_{k}^{*}\left(\hbar \omega-\widetilde{E}_{\downarrow}(k)+\varepsilon_{k}\right)\left\langle d_{k \uparrow}^{+} a_{k \uparrow}\right\rangle+\right. \\
& \left.\left.+V_{k}\left\langle a_{k \downarrow}^{+} d_{k \downarrow}\right\rangle\left(\hbar \omega+\widetilde{E}_{\uparrow}(k)-\varepsilon_{k}\right)\right]\right\}\left\{-\left|V_{k}\right|^{2}(2 \hbar \omega+\Delta)^{2}+\right. \\
& \left.+\hbar \omega\left(\hbar \omega+\widetilde{E}_{\uparrow}(k)-\widetilde{E}_{\downarrow}(k)\right)\left(\hbar \omega-\widetilde{E}_{\downarrow}(k)+\varepsilon_{k}\right) \times\left(\hbar \omega+\widetilde{E}_{\uparrow}(k)-\varepsilon_{k}\right)\right\}^{-1},
\end{aligned}
$$

which is satisfied if $\hbar \omega_{0}=0$. It follows from general considerations that when the wave length of a spin wave is long, its energy $\hbar \omega_{q}$ must be related to the wave number $q$ by $\hbar \omega=D q^{2}$ [17]. The exact formula for $D$ valid for any metallic or non-metallic ferromagnet, or for a non-ferromagnetic material in a static magnetic field is [17].

$$
D q^{2}=\frac{1}{2\left\langle S^{z}\right\rangle}\left\{\hbar q\left\langle\left[J_{q}^{-}, S_{-q}^{+}\right]\right\rangle-\hbar^{2} q^{2} \lim _{\omega \rightarrow 0} \lim _{q \rightarrow 0} X_{J}\right\} .
$$

(For the definitions see Ref. 17.) Thus the solution for the equation

$$
1=U /\left(g \mu_{\mathrm{B}}\right)^{2} X^{\mathrm{HF}}(q, \omega)
$$

exists which has the property $\lim \hbar \omega_{q}=0$ and this solution corresponds to a spin-wave excitation in the model with $s-d$ hybridization (2.1).

Thus, we have derived a formula (3.8) for the dynamical susceptibility $X^{-}(q, \omega)$ in RPA and have shown, that it can be calculated in terms of the Hartree-Fock response function $X^{H F}(q, \omega)$ analogous to the Izuyama et al. [1] expression.

\section{The Hartree-Fock Susceptibility}

To calculate the Stoner-mode cross section, we need the poles of the HartreeFock susceptibility $X^{\mathrm{HF}}(q, \omega)$ of Eq. (3.9). If we note that the susceptibility $X^{H F}(q, \omega)$ corresponds to the susceptibility of the system which is described by the following Hamiltonian

$$
H^{\mathrm{HF}}=\sum_{k, \sigma} \widetilde{E}_{\sigma}(k) d_{k \sigma}^{+} d_{k \sigma}+\sum_{k, \sigma} \varepsilon_{k} a_{k \sigma}^{+} a_{k \sigma}+\sum_{k \sigma}\left(V_{k} a_{k \sigma}^{+} d_{k \sigma}+V_{k}^{*} d_{k \sigma}^{+} a_{k \sigma}\right)
$$

that the poles of $X^{\mathbf{H F}}(q, \omega)$ may be simply calculated. The Hamiltonian (4.1) may be diagonalized by the canonical $(u, v)$-transformation. The result is $[11,16]$

$$
H^{\mathrm{HF}}=\sum_{k \sigma}\left\{\omega_{1 k \sigma} \alpha_{k \sigma}^{+} \alpha_{k \sigma}+\omega_{2 k \sigma} \beta_{k \sigma}^{+} \beta_{k \sigma}\right\}
$$

where

$$
\begin{aligned}
& \omega_{2 k \sigma}^{1}=\frac{1}{2}\left\{\left(\tilde{E}_{\sigma}(k)+\varepsilon_{k}\right) \pm \sqrt{\left(\tilde{E}_{\sigma}(k)-\varepsilon_{k}\right)^{2}+4\left|V_{k}\right|^{2}}\right\} \\
& \left.\begin{array}{c}
u_{k \sigma}^{2} \\
v_{k \sigma}^{2}
\end{array}\right\}=\left[1+\frac{\left(\omega_{2 k \sigma}^{1}-\widetilde{E}_{\sigma}(k)\right)^{2}}{\left(V_{k}^{*}\right)^{2}}\right]^{-1} .
\end{aligned}
$$


The susceptibility can be evaluated to be

$$
\begin{aligned}
& X^{\mathrm{HF}}(q, \omega)=\left(g \mu_{\mathrm{B}}\right)^{2} / N \sum_{k}\left\{u_{k+q \downarrow}^{2} u_{k \uparrow}^{2} \frac{n_{k \uparrow}^{\alpha}-n_{k+q \downarrow}^{\alpha}}{\hbar \omega+\omega_{1 k+q \downarrow}-\omega_{1 k \uparrow}}+\right. \\
& +v_{k+q \downarrow}^{2} v_{k \uparrow}^{2} \frac{n_{k \uparrow}^{\beta}-n_{k+q \downarrow}^{\beta}}{\hbar \omega+\omega_{2 k+q \downarrow}-\omega_{2 k \uparrow}}+u_{k+q \downarrow}^{2} v_{k \uparrow \uparrow}^{2} \frac{n_{k \uparrow}^{\beta}-n_{k+q \downarrow}^{\alpha}}{\hbar \omega+\omega_{1 k+q \downarrow}-\omega_{2 k \uparrow}}+ \\
& \left.+v_{k+q \downarrow}^{2} u_{k \uparrow}^{2} \frac{n_{k \uparrow}^{\alpha}-n_{k+q \downarrow}^{\beta}}{\hbar \omega+\omega_{2 k+q \downarrow}-\omega_{1 k \uparrow}}\right\} \text {. }
\end{aligned}
$$

From Eq. (4.5) one can obtain the usual form of the Stoner-type condition for ferromagnetism [18] at $T=0^{\circ} \mathrm{K}$

$$
1=U /\left(g \mu_{\mathrm{B}}\right)^{2} X^{\mathrm{HF}}(0,0)=U \cdot \varrho_{\mathrm{eff}}\left(\varepsilon_{F}\right) .
$$

Turning now to the calculation of the cross section (2.8), we obtain from Eq. (3.8) the imaginary part of $X^{-}(q, \omega)$ namely,

$\operatorname{Im} X^{-}(q, \omega)=\operatorname{Im} X^{H F}(q, \omega)\left\{\left[1-U /\left(g \mu_{B}\right)^{2} \operatorname{Re} X^{H F}(q, \omega)\right]^{2}+\right.$

$$
\left.+\left[U /\left(g \mu_{\mathrm{B}}\right)^{2} \operatorname{Im} X^{\mathrm{HF}}(q, \omega)\right]^{2}\right\}^{-1} .
$$

From (4.5) we obtain for $\operatorname{Im} X^{H F}(q, \omega)$ the result

$$
\begin{aligned}
\operatorname{Im} X^{H F}(q, \omega)= & -\pi\left(g \mu_{\mathbf{B}}\right)^{2} 1 / N \sum_{k}\left\{u_{k+q \downarrow}^{2} u_{k \uparrow}^{2}\left(n_{k \uparrow}^{\alpha}-n_{k+q \downarrow}^{\alpha}\right) \delta\left(\hbar \omega+\omega_{1 k+q \downarrow}-\omega_{1 k \uparrow}\right)+\right. \\
& +v_{k+q \downarrow}^{2} v_{k \uparrow}^{2}\left(n_{k \uparrow}^{\beta}-n_{k+q \downarrow}^{\beta}\right) \delta\left(\hbar \omega+\omega_{2 k+q \downarrow}-\omega_{2 k \uparrow}\right)+ \\
& +u_{k+q \downarrow}^{2} v_{k \uparrow}^{2}\left(n_{k \uparrow}^{\beta}-n_{k+q \downarrow}^{\alpha}\right) \delta\left(\hbar \omega+\omega_{1 k+q \downarrow}-\omega_{2 k \uparrow}\right)+ \\
& \left.+v_{k+q \downarrow}^{2} u_{k \uparrow}^{2}\left(n_{k \uparrow}^{\alpha}-n_{k+q \downarrow}^{\beta}\right) \delta\left(\hbar \omega+\omega_{1 k+q \downarrow}-\omega_{2 k \uparrow}\right)\right\} .
\end{aligned}
$$

Now it follows from $\mathrm{Eq} .(4.7)$ that $\operatorname{Im} X^{\mathrm{HF}}(q, \omega)$ is non-zero only for values of $\hbar \omega$ equal to the energies of the Stoner-type excitations

$$
\begin{aligned}
& \hbar \omega_{1}=\omega_{1 k \uparrow}-\omega_{1 k+q \downarrow}, \\
& \hbar \omega_{2}=\omega_{2 k \uparrow}-\omega_{2 k+q \downarrow}, \\
& \hbar \omega_{3}=\omega_{2 k \uparrow}-\omega_{1 k+q \downarrow}, \\
& \hbar \omega_{4}=\omega_{1 k \uparrow}-\omega_{2 k+q \downarrow} .
\end{aligned}
$$

The spin wave pole occurs where $\operatorname{Im} X^{H F}(q, \omega)$ tends to zero. In this case, we can in (4.6) take the limit $\operatorname{Im} X^{\mathrm{HF}}(q, \omega) \rightarrow 0$ so that

$$
U /\left(g \mu_{\mathrm{B}}\right)^{2} \operatorname{Im} X^{-}(q, \omega)=-\pi \delta\left\{1-U /\left(g \mu_{\mathrm{B}}\right)^{2} \operatorname{Re} X^{\mathrm{HF}}(q, \omega)\right\} .
$$

But

$$
1-U /\left(g \mu_{B}\right)^{2} \operatorname{Re} X^{H F}(q \rightarrow 0, \omega \rightarrow 0) \sim b^{-1}\left(\hbar \omega-\hbar \Omega_{q}\right)
$$

and thus

$$
\operatorname{Im} X^{-}(q \rightarrow 0, \omega \rightarrow 0) \sim-\pi\left(g \mu_{\mathrm{B}}\right)^{2} b / U \delta\left(\hbar \omega-\hbar \Omega_{q}\right)
$$

Here $b$ is a certain constant, which can be numerically calculated and $\hbar \Omega_{q}$ is the acoustical spin wave pole $\hbar \Omega_{q \rightarrow 0}=0$. Using (2.8) with (4.11) leads to the 
expression

$$
\begin{aligned}
\left(\frac{d^{2} \sigma}{d \Omega d E^{\prime}}\right)_{\mathrm{tr.}}= & \left(\frac{\gamma e^{2}}{m_{e} c^{2}}\right)^{2} \frac{1}{4}|F(q)|^{2} \frac{k^{\prime}}{k}\left(1+\tilde{q}_{z}^{2}\right) N \frac{b}{U} \times \\
& \times \sum_{p}\left[n\left(\Omega_{p}\right) \delta\left(\hbar \omega+\hbar \Omega_{p}\right)+\left(1+n\left(\Omega_{p}\right)\right) \delta\left(\hbar \omega-\hbar \Omega_{p}\right)\right] .
\end{aligned}
$$

The cross section (4.12) does not include the contribution arising from the scattering by Stoner excitation; i.e. that determined by $X^{\mathrm{HF}}(q, \omega)$. It was shown by Thompson [19] that in a single-band Hubbard-Hamiltonian model of transition metal in the limit when the wave vector of the elementary excitations goes to zero, the acoustic spin-wave mode dominates the inelastic neutron scattering, and the contribution to the cross section due to the Stoner-mode scattering goes to zero. Thompson showed that the Stoner-mode scattering intensity does not become comparable to the spin-wave scattering intensity until $q=0.9 q_{\max }$ (the value of $q$ when the spin wave enters the continuum). For large values of $q$ and $\omega$ the energy gap for spin flipping Stoner excitations may be overcome.

In this case

$$
\operatorname{Im} X^{-}(q, \omega) \approx \operatorname{Im} X^{H F}(q, \omega)
$$

With (2.8) and (4.13) we obtain

$$
\begin{aligned}
\left(\frac{d^{2} \sigma}{d \Omega d E^{\prime}}\right)_{\mathrm{tr} .}= & \left(\frac{\gamma e^{2}}{m_{e} c^{2}}\right)^{2} \frac{1}{4}|F(q)|^{2} \frac{k^{\prime}}{k}\left(1+\tilde{q}_{z}^{2}\right) \frac{N}{\pi\left(g \mu_{\mathrm{B}}\right)^{2}} \times \\
& \times\left\{(n(\omega)+1) \operatorname{Im} X^{\mathrm{HF}}(-q, \omega)+n(-\omega) \operatorname{Im} X^{\mathrm{HF}}(q, \omega)\right\} .
\end{aligned}
$$

Although in Thompson's model the Stoner-mode scattering cross section remains small until $q$ is fairly close to $q_{\max }$, it will be shown by Sokoloff [9] that in the system of two degenerate parabolic bands the Stoner-mode cross section may become large for much smaller scattering vector. The region of the intersection of the spin wave spectrum with a continuum band of Stoner excitations was achieved in a recent work [7] by a high flux reactor.

\section{Conclusion}

The essential result of the present paper is the extension of Izuyama et al. [1] treatment for the model with $s-d$ hybridization which is more realistic for $3 d$ transition metals than the single-band Hubbard-Hamiltonian model. The present qualitative consideration shows that a two-band picture of low-temperature inelastic neutron scattering is modified in comparison with the single-band HubbardHamiltonian model. We have found that the long-wave-length acoustic spin wave excitations should exist in this model and that in the limit $q \rightarrow 0$, the acoustic spin-wave mode dominates the inelastic neutron scattering. The spin-wave part of the cross section is renormalized only quantitatively. The cross section due to Stoner-mode scattering is qualitatively modified because of occurring of the four Stoner-type bands which may lead to the modification of the spin wave intensity fall off with increasing energy [19]. The intersection point $q_{\max }$ must be strongly renormalized. The concrete neutron inelastic scattering picture is strongly energyband-structure dependent and must be numerically investigated. 
In our treatment of the correlation problem we have used the random phase approximation. This approximation is well applied for a weakly correlated system, whilst in the derivation of the cross section (2.5) it is essentially assumed that the overlap of the Wannier functions is negligible. Thus in this scheme

where

$$
S^{ \pm}(q, t)=F^{\prime}(q) \tilde{s}^{ \pm}(q, t)
$$

$$
F(q)=\int d^{3} r e^{i q r}\left|\varphi_{d}\right|^{2}
$$

is the geometrical distributions or the form factor. Also, when $U$ is large the theory becomes in many respects similar to that of a hard sphere Fermi gas, in which the effective interaction is measured by the scattering length rather than the potential scattering [20]. Because of this the RPA encounters serious difficulties when the interaction $U$ becomes strong. These difficulties have been discussed within the $t$-matrix approximation [20], which reduces, in the appropriate limit, to the RPA result. A more interesting method has recently been proposed by Sakurai [21] and has been used by Kikoin [9] for the Hubbard model with orbital degeneracy. This method does not reduce to the RPA results.

Therefore, it seems interesting to investigate our model Hamiltonian (2.1) by the Sakurai method so as to be able to calculate the transverse and longitudinal neutron inelastic cross sections in a strongly correlated problem. We hope to be able to do this in the near future.

Acknowledgements. The present work has been started on the suggestion of Dr. L. Dobrzynski. The author would like to thank him for useful discussions.

It is a great pleasure to thank Professor Konrad Fischer, Doctors J. Solyom, K. Elk for helpful and illuminating discussions. Thanks are also due to Doctors N. M. Plakida, K. A. Kikoin and L. A. Maksimov for their interesting comments.

\section{References}

1. Marshall, W., Lovesey, S. W.: Theory of thermal neutron scattering. Oxford: Clarendon Press 1971

2. Lowde, R. D., Windsor, C. G.: Adv. Phys. 19, 813 (1970)

3. Pickart, S. J., Alperin, H. A., et al.: Phys. Rev. 156, 623 (1967)

4. Shirane, G., Minkiewicz, V. J., Nathaus, R.: J. appl. Phys. 39, 383 (1968)

5. Mook, H. A., Nicklow, R. M., et al.: J. appl. Phys. 40, 1450 (1969)

6. Thompson, E. D., Mook, H. A.: J. appl. Phys. 41, 1227 (1970)

7. Mook, H. A., Nicklow, R. M.: Phys. Rev. B 7, 336 (1973)

8. Thompson, E. D.: Adv. Phys. 14, 213 (1965)

9. Yamada, H., Shimizu, M.: J. Phys. Soc. Japan 22, 1404 (1967) ; 25, 1001 (1968) Sokoloff, J. B.: Phys. Rev. 180, 613 (1969)

Kikoin, K. A.: Fiz. Trerd. Tela 14, 1329 (1972)

10. George, P. K.: Physica 49, 278 (1970)

Schneider, J., Heiner, E., Haubenreisser, W.: Phys. stat. solidi 52, K17 (1972)

Plakida, N. M., Smirnov, L. S.: JINR, P 4-7371, 1973

11. Monohar, C.: Sol. St. Commun. 9, 2025 (1971)

12. Kishore, R., Joshi, S. K.: Phys. Rev. B3, 3901 (1971)

Schneider, J., Heiner, E., Haubenreisser, W.: Phys. status solidi 54, 577 (1972)

Heiner, E., Schneider, J.: Phys. status solidi 55, 93 (1973)

13. Cooke, J. F.: Phys. Rev. B 7, 1108 (1973)

14. Kishore, R., Joshi, S. K.: Phys. Rev. B2, 1411 (1970)

15. Elk, K.: Phys. status solidi 48, K 93 (1971); JINR, E4-7030, Dubna, 1973

16. Kuzemsky, A. L.: JINR, P4-7749, Dubna, 1974 
17. Edwards, D. M., Fisher, B.: J. Physique 32, C1-697 (1971)

18. Fischer, K.: Phys. status solidi 46, 11 (1971)

19. Thompson, E. D.: Phys. Rev. Letters 19, 635 (1967)

20. Young, W., Callaway, J.: J. Phys. Chem. Solids 31, 865 (1970)

21. Sakurai, A.: Progr. theor. Phys. 39, 312 (1968)

Dr. A. L. Kuzemsky

Laboratory of Theoretical Physics

Joint Institute for Nuclear Research

Head Post Office P.0. Box 79

Moscow USSR 